\title{
A NORM CONDITION FOR DISCONJUGACY OF COMPLEX DIFFERENTIAL SYSTEMS
}

\author{
SHUI-NEE CHOW ${ }^{1}$ AND PUI-KEI WONG
}

ABstract. A first order linear vector differential equation with coefficients holomorphic in the unit disk is considered. A criterion for disconjugacy expressed in terms of the Euclidean norm is given, and the condition is the best possible for this particular norm.

In this note we are concerned with complex vector differential equations of the form

$$
w^{\prime}(z)=A(z) w(z),
$$

where $A(z)=\left(A_{j k}(z)\right)$ is a given $n \times n$ matrix whose entries are functions holomorphic in the unit disk $D=\{z:|z|<1\}$ and $w(z)=\left(w_{1}(z), \cdots, w_{n}(z)\right)$. The equation (1) is called $S$-disconjugate in $D$ if, for every choice of $n$ points $\alpha_{1}, \cdots, \alpha_{n}$ in $D$, the only solution $w$ for which $w_{j}\left(\alpha_{j}\right)=0, j=$ $1, \cdots, n$, is the trivial solution, cf. [6].

In [7] Schwarz showed that if $\|A\|_{\infty}$ is the maximum-row norm of the matrix $A$ and if, for every distinct pair of points $z_{0}$ and $z$ joined by a path in $D$,

$$
\int_{z_{0}}^{z}\|A(z)\|_{\infty}|d z|<2 \log 2,
$$

then (1) is $S$-disconjugate in $D$. Recently, he further showed in [8] that condition (2) may be replaced by

$$
\int\|A(\zeta)\||d \zeta| \leqq 2
$$

where $C$ is the unit circle $|\zeta|=1$ and $\|A\|$ is any matrix norm consistent with an absolute vector norm $\|w\|$. Moreover, he observed that it is not possible to replace the constant 2 in (3) by any number greater than $\pi$ (see [8, p. 321]). In this note we shall show that in the case of the Euclidean norm the constant 2 above can in fact be replaced by $\pi$. For systems of a real interval the corresponding result has been obtained by Nehari [5]. For other criteria of $S$-disconjugacy see [4], [6], [7], and [8].

Received by the editors November 22, 1971.

AMS 1970 subject classifications. Primary 34A20; Secondary 34C10.

Key words and phrases. Complex differential systems, disconjugate solutions.

${ }^{1}$ Partially supported under NSF grant GU-2648. 
Suppose $\|w\|$ is the Euclidean norm of $w$ defined by

$$
\|w\|=\langle w, \bar{w}\rangle^{1 / 2}=\left(\sum_{j=1}^{n}\left|w_{j}\right|^{2}\right)^{1 / 2}
$$

then it is well known that the square root of the maximal characteristic value of $A A^{*}$ is a norm for $A$ consistent with $\|w\|$. Denote this norm also by $\|A\|$.

THEOREM. If

$$
\int\|A(\zeta)\||d \zeta| \leqq \pi
$$

then (1) is $S$-disconjugate in $D$.

The proof of this result will require four lemmas which we state below:

LEMMA 1 (CARLSON [1]). Let $B$ be a complex Banach space with norm $\|\cdot\|$ and let $f: D \rightarrow B$ be holomorphic in $D$. Suppose $\Gamma$ is a rectifiable arc which lies in $D$ and $\zeta$ is any point on the circle $|\zeta|=r, 0<r<1$, which contains $\Gamma$ in its interior. Denote by

$$
V_{\Gamma}(\zeta)=\int_{\Gamma}|d[\arg (\zeta-z)]|, \quad z \in \Gamma .
$$

Then for any $p>0$,

$$
\int\|f(z)\|^{p}|d z| \leqq \lim _{r \uparrow 1} \frac{1}{\pi} \int_{0}^{2 \pi}\left\|f\left(r e^{i \theta}\right)\right\|^{p} V_{\Gamma}\left(r e^{i \theta}\right) d \theta,
$$

whenever the right-hand side has meaning.

LEMMA 2 (KIM [2]). Suppose (1) is not $S$-disconjugate in D. Then there exist $n$ points $\alpha_{1}, \cdots, \alpha_{n}$ with $\left|\alpha_{j}\right|=K, 0<K<1$, and a nontrivial solution w such that $w_{j}\left(\alpha_{j}\right)=0, j=1, \cdots, n$.

LEMMA 3. If $P_{1}, \cdots, P_{m}$ are $m$ points on the unit sphere $S$ of $E^{m}$, $P_{i}=\left(P_{i 1}, \cdots, P_{i m}\right), P_{i i}=0, i=1, \cdots, m$. Then

$$
\sum_{i=1}^{m} \rho\left(P_{i}, P_{i+1}\right) \geqq \pi,
$$

where $\rho(x, y)$ is the length of the geodesic arc joining $x$ with $y$ on $S$ and $P_{m+1}=P_{1}$.

LEMMA 4. Let $w$ be an absolutely continuous vector field on $D$ such that $\|w(z)\|>0$ for all $z \in D$. If

$$
\mu(z)=\|w(z)\|^{-\mathbf{1}} w(z),
$$


then

$$
\left\|\mu^{\prime}(z)\right\| \leqq\|w(z)\|^{-1}\left\|w^{\prime}(z)\right\| .
$$

We first remark that Lemma 1 was proved by F. Carlson [1] with $B$ being the complex number field, but his proof is equally valid for any complex normed linear space. Lemma 2 of Kim [2] has been used by Schwarz ([7], [8]) to generate criteria of $S$-disconjugacy. Lemma 3 is an extension of a geometrical lemma of Lasota and Olech [3] and Zaks [10] has recently given a very simple proof of this result.

Turning now to the proof of Lemma 4 we first note that

$$
\begin{aligned}
u(z) & =\frac{1}{2}\|w(z)\|(d / d z)\|w(z)\| \\
& =\frac{1}{2}(d / d z)\langle w(z),(w(z))-\rangle \\
& =\frac{1}{2}\left[\left\langle w^{\prime}(z),(w(z))-\right\rangle+\left\langle w(z),\left(w^{\prime}(z)\right)-\right\rangle\right] .
\end{aligned}
$$

It follows from (6) and (8) that

$$
\begin{aligned}
\left\|\mu^{\prime}(z)\right\| & =\left\|\left\{w^{\prime}(z)\|w(z)\|^{-1}-w(z)\|w(z)\|^{-2}(d / d z)\|w(z)\|\right\}\right\| \\
& =\left\|\left\{w^{\prime}(z)\|w(z)\|^{-1}-u(z) w(z)\|w(z)\|^{-3}\right\}\right\| \\
& =\left[\left\|w^{\prime}(z)\right\|^{2}\|w(z)\|^{2}-u^{2}(z)\right]^{1 / 2}\|w(z)\|^{-2}
\end{aligned}
$$

Since $0 \leqq\left\|w^{\prime}(z)\right\|^{2}\|w(z)\|^{2}-u^{2}(z) \leqq\left\|w^{\prime}(z)\right\|^{2}\|w(z)\|^{2}$, (7) follows immediately.

To prove the theorem we suppose the contrary conclusion so that by Lemma 2 , there exist $n$ points $\alpha_{1}, \cdots, \alpha_{n}$ on a circle $C_{K}=\{z:|z|=K$, $0<K<1\}$ and a nontrivial solution $w$ such that $w_{j}\left(\alpha_{j}\right)=0, j=1, \cdots, n$. The function $\mu(z)$ defined by (6) is then absolutely continuous and of norm 1 on $C_{K}$ such that $\mu_{j}\left(\alpha_{j}\right)=0, j=1, \cdots, n$. The image of $C_{K}$ may then be regarded as a closed path on the unit sphere in $E^{2 n}$ so that Zak's Lemma together with equation (1) yields

$$
\pi \leqq C_{C_{K}} \int\left\|\mu^{\prime}(z)\right\||d z| \leqq C_{C_{R}} \int\|A(z)\||d z| .
$$

On the other hand, if $\zeta$ is any point on the unit circle $C$ then the total angular variation subtended by $C_{K}$ with respect to $\zeta$ is clearly twice that

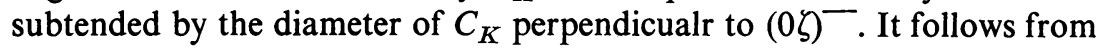
elementary considerations that

$$
V_{C_{K}}(\zeta) \leqq 4 \tan ^{-1} K, \quad \zeta \in C .
$$

Putting this estimate into (4) and using the hypothesis, we find that

$$
{ }_{C_{K}} \int\|A(z)\||d z| \leqq \frac{4}{\pi} \tan ^{-1} K{ }_{C} \int\|A(\zeta)\||d \zeta| \leqq 4 \tan ^{-1} K .
$$


Combining this with (9) we arrive at

$$
\frac{\pi}{4} \leqq \tan ^{-1} K, \quad 0<K<1,
$$

which is clearly impossible. This contradiction proves the theorem.

Corollary. Suppose $p \geqq 1$ and

$$
\frac{1}{\pi}_{C} \int\|A(\zeta)\|^{p}|d \zeta| \leqq\left(\frac{2}{\pi}\right)^{p-1} ;
$$

then (1) is $S$-disconjugate in $D$.

To prove this we proceed as in the proof of the theorem and apply the Hölder inequality to the last integral in (9). Then, for $0<K<1$,

$$
\pi \leqq\left(C_{C_{K}} \int\|A(z)\|^{p}|d z|\right)^{1 / p}(2 \pi K)^{1 / q},
$$

where $(1 / p)+(1 / q)=1$. As before, Carlson's inequality yields

$$
C_{K} \int\|A(z)\|^{p}|d z| \leqq \frac{4}{\pi} \tan ^{-1} K_{C} \int\|A(\zeta)\|^{p}|d \zeta| .
$$

Combining this with (10) and (11) we find that

$$
\pi \leqq 4 K^{1 / q}(\arctan K)^{1 / p}, \quad 0<K<1,
$$

which is the desired contradiction.

We remark that for second order linear equations of the form

$$
\left[w^{\prime}(z) / k(z)\right]^{\prime}+q(z) w(z)=0,
$$

$S$-disconjugacy is also known as disfocality. In this case

$$
A=\left(\begin{array}{cc}
0 & k(z) \\
q(z) & 0
\end{array}\right)
$$

so that $\|A(z)\|=\max (|k(z)|,|q(z)|)$. According to (11), a sufficient condition for (12) to be disfocal in $D$ is that

$$
\frac{1}{\pi_{C}} \int[\max (|k|,|q|)]^{p}|d \zeta| \leqq\left(\frac{2}{\pi}\right)^{p-1}
$$

for some $p \geqq 1$. For a different norm criterion for disfocality of (12) see [9].

In conclusion we shall consider an example of a two-dimensional system on the real interval $[0, \pi / 2]$ of the form $x^{\prime}(t)=A(t) x(t)$, where

$$
A=\left(\begin{array}{rr}
0 & 1 \\
-1 & 0
\end{array}\right)
$$


If $x=\left(x_{1}, x_{2}\right)$ and we take as norm $\|x\|_{\infty}=\max \left(\left|x_{1}\right|,\left|x_{2}\right|\right)$, then $\|A(t)\|_{\infty}=2$. In this case a solution is

$$
x(t)=(\sin t, \cos t),
$$

and a simple computation shows that $\left\|x^{\prime}(t)\right\|_{\infty}=\|x(t)\|_{\infty}$ on $[0, \pi / 2]$. Moreover, if $\mu(t)$ is defined as in (6), then

$$
\begin{aligned}
\left\|\mu^{\prime}(t)\right\|_{\infty} & =1+\tan ^{2} t, & & 0 \leqq t \leqq \pi / 4, \\
& =1+\cot t, & & \pi / 4 \leqq t \leqq \pi / 2 .
\end{aligned}
$$

It follows that $\left\|\mu^{\prime}(t)\right\|_{\infty} \geqq 1=\|x(t)\|_{\infty}^{-1}\left\|x^{\prime}(t)\right\|_{\infty}$ and Lemma 4 is therefore not valid for this norm.

ADDED IN PROOF. The authors have just learned that Professor B. Schwarz has also obtained the result of our theorem using similar techniques. For details, see his paper Curves on the unit sphere and disconjugacy of differential systems, J. Math. Anal. Appl. (to appear).

\section{REFERENCES}

1. F. Carlson, Quelques inégalites concernant les fonctions analytiques, Ark. Mat. Astr. Fys. 29B (1943), no. 11, 6 pp. MR 6, 205.

2. W. J. Kim, Disconjugacy and disfocality of differential systems, J. Math. Anal. Appl. 26 (1969), 9-19. MR 38 \#4759.

3. A. Lasota and C. Olech, An optimal solution of Nicoletti's boundary value problem, Ann. Polon. Math. 18 (1966), 131-139. MR 34 \#4580.

4. D. London and B. Schwarz, Disconjugacy of complex differential systems and equations, Trans. Amer. Math. Soc. 135 (1969), 487-505. MR 38 \#6154.

5. Zeev Nehari, Oscillation theorems for systems of linear differential equations, Trans. Amer. Math. Soc. 139 (1969), 339-347. MR 39 \#542.

6. Binyamin Schwarz, Disconjugacy of complex differential systems, Trans. Amer. Math. Soc. 125 (1966), 482-496. MR 34 \#6190.

7. - Norm conditions for disconjugacy of complex differential systems, J. Math. Anal. Appl. 28 (1969), 553-568. MR 40 \#2963.

8. - Mappings of domains of components of solutions of differential systems, J. Differential Equations 10 (1971), 314-323.

9. Pui-Kei Wong, A disfocality criterion, Proc. Amer. Math. Soc. 30 (1971), 112-114.

10. Joseph Zaks, On the length of some arcs in the unit sphere (to appear).

Department of Mathematics, Michigan State University, East Lansing, MiCHIGAN 48823 